\title{
Asporin Is Highly Expressed in CAFs of High Grade Serous Ovarian Cancinoma Patients and Predicts Poor Prognosis
}

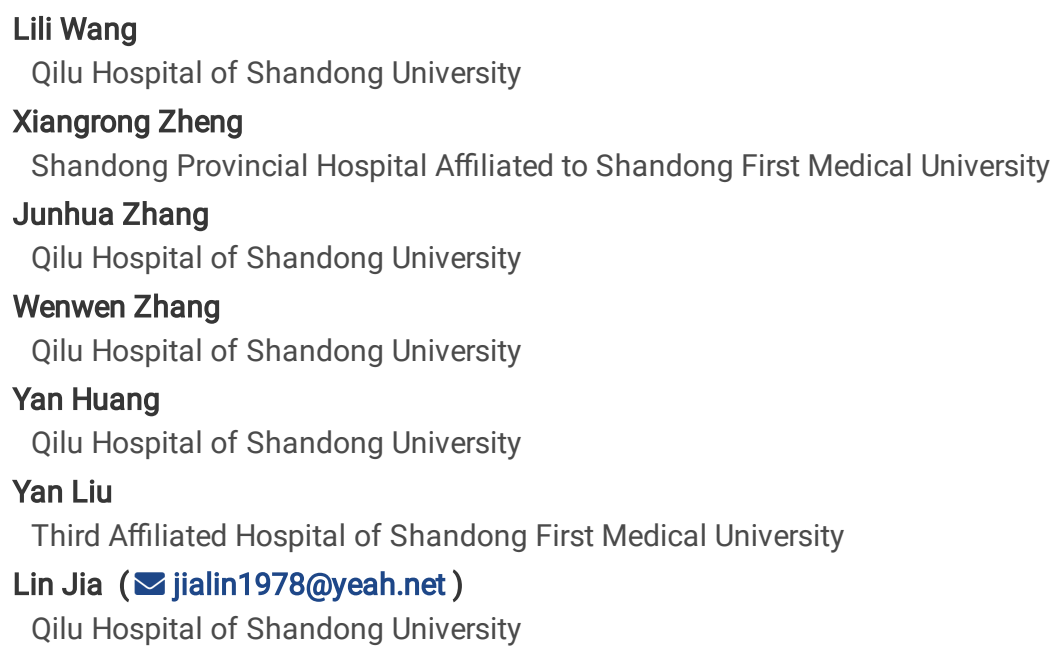

Research

Keywords: High grade serous ovarian cancinoma(HGSOC), Asporin, CD44, prognosis

Posted Date: October 29th, 2021

DOI: https://doi.org/10.21203/rs.3.rs-1012672/v1

License: (c) (i) This work is licensed under a Creative Commons Attribution 4.0 International License. Read Full License 


\section{Abstract}

Background: Tumor microenvironment is widely considered as a critical factor influencing prognosis of ovarian cancer patients. To further improve the unsatisfied clinical outcome of ovarian cancer patients, it is desperately needed to explore biomarkers with potential clinical value of ovarian cancer. Asporin (ASPN), as a member of the proteoglycan family in tumor microenvironment, has been proved to play a vital role in cancer progression by binding with CD44 receptor. However, there is still no report about the research of Asporin in ovarian cancer. The purpose of the present study was to explore the expression and prognostic significance of Asporin in high grade serous ovarian cancinoma(HGSOC).

Methods: The expression level of Asporin and CD44 in 85 formalin fixed paraffin embedded (FFPE) samples of 85 HGSOC patients was detected by immunohistochemistry. The association between the expression of the two biomarkers and the clinicopathologic factors of HGSOC was assessed by $\chi^{2}$ test. The survival analyses were performed by Kaplan-Meier.

Results: Asporin was mainly expressed in CAFs of 85 HGSOC patients, while CD44 was mainly expressed on membrane of tumor cells. The positive rates of Asporin, CD44, and Asporin /CD44 co-expression were 43.53\% (37/85), 34.12\% (29/85) and 28.23\% (24/85) in 85 HGSOC tissues, respectively. There were close associations between Asporin, CD44, Asporin/CD44 expression and CA125 level of blood, tumor differentiation, FIGO staging, chemoresistance of HGSOC patients. Further, univariate survival analysis showed that positive expression of Asporin and positive Asporin/CD44 co-expression of HGSOC indicated poor clinical outcomes, and multivariate survival analysis suggested that positive expression of Asporin and positive Asporin/CD44 co-expression were independent prognostic factors for disease-free survival (DFS) and overall survival (OS) of HGSOC patients, respectively.

Conclusion: Asporin was mainly expressed in CAFs of HGSOC patients. High expression of Asporin may identify a subgroup of HGSOC patients with more chemoresistance clinicopathological features and may be a potential prognostic predictor and therapeutic target for HGSOC patients.

\section{Background}

Ovarian cancer is one of the most deadly malignancies in females worldwide. More than $70 \%$ of ovarian cancer patients are diagnosed as advanced stage, with extensive invasion and metastasis [1]. In 2021, the American Cancer Society (ASC) reported that the number of deaths caused by ovarian cancer ranked fifth among all cancers[2]. High grade serous ovarian cancer (HGSOC) is the most common pathological subtype of ovarian cancer. Tumor cytoreductive surgery combined platinum-based chemotherapy regimens is the first-line treatment option for HGSOC patients[1]. Frustratingly, 80-90\% HGSOC patients will turned to be drug-resistance during chemotherapy, with a 5-year survival rate less than $30 \%[2]$. Research on chemotherapy resistance of ovarian cancer has been carried out for many years. However, the molecular mechanism of chemotherapy resistance of ovarian cancer has not been clarified. Therefore, further to elucidate the chemotherapy resistance mechanism of HGSOC is of great significance to reduce chemotherapy resistance and improve the prognosis of HGSOC patients. Our group has focused on chemo-resistance of ovarian cancer for many years[3-5]. Recent years, the role of tumor microenvironment in chemoresistance of ovarian cancer has aroused our interest.

Tumor microenvironment was proposed by Lord in 1979 and mainly composed of immune cells, interstitial cells, extracellular matrix (ECM), cytokines and other components[6]. It is well known that the heterogeneity, dynamics and remodeling of tumor microenvironment not only create various favorable conditions for tumor growth, invasion and metastasis, but also play a pivotal role in inducing tumor cells to acquire drug resistance[7]. In recent years, the published studies in ovarian cancer demonstrated that the tumor microenvironment of ovarian cancer could promote the drug resistance of ovarian cancer by influencing and maintaining the unique phenotype and malignant behavior of cancer cells. On the contrary, cancer cells regulate and stimulate microenvironmental components to differentiate into pro-tumor phenotypes in the process of progression, further promote the progression of ovarian cancer, and mediate immune escape and chemotherapy resistance [8]. So, it is of great potential to improve the prognosis of ovarian cancer patients by regulating the components of ovarian cancer microenvironment to reduce ovarian cancer chemotherapy resistance. Cancer-Associated-Fibroblasts (CAFs), as one of the main components in the tumor microenvironment of ovarian cancer, play an important role in mediating chemotherapy resistance of ovarian cancer by secreting a large number of cytokines, chemokines, growth factors and receptors [9-12]. The previously published study in pancreatic cancer of our group demonstrated that CAFsderived Asporin, as a newly discovered ECM protein, could promote invasion and metastasis of cancer cells [13]. In addition, the results also showed that high expression of Asporin indicated poor prognosis of patients[13]. However, thus far, the expression and biological role of Asporin in ovarian cancer has not been reported. This study aimed to explore the expression and the clinical value of the Asporin in HGSOC patients and to provide promising biological target for overcoming the chemoresistance of HGSOC.

\section{Materials And Methods}

\section{Patients and clinicopathologic data}

Formalin fixed paraffin embedding (FFPE) samples from 85 HGSOC patients, including 53 platinum resistance and 32 platinum sensitive HGSOC patients, from Qilu Hospital of Shandong University between January 2010 and January 2015 were included in the present study according to the

Page 2/13 
following inclusion criteria. 1) HGSOC patients were firstly diagnosed and had surgical treatment in Qilu Hospital of Shandong University, finally confirmed by postoperative histopathology. 2) HGSOC patients with satisfactory cytoreductive surgery. 3) HGSOC patients with complete clinicopathological and follow-up data, which was obtained from Gynecology Oncology Key Laboratory, Qilu Hospital of Shandong University. 4) Hematoxylin and eosin stained slides of all samples were reconfirmed by two experienced histopathologists. The FFPE specimens were preserved at room temperature.

\section{Antibodies and main reagents}

Anti-Asporin (Abcam, 1:200) and anti-CD44 (Cell Signaling Technology, 1:200); rabbit anti-mouse-Alexa Fluor (1:100; Life Technologies, USA) and goat anti-rabbit-FITC (1:100; Life Technologies, USA); Fluoromount G media with DAPI nuclear stain (Santa Cruz Biotechnology, CA, USA)

\section{Immunohistochemistry staining}

Representative blocks of high grade serous ovarian cancer (HGSOC) tissues were selected by two experienced pathologists via hematoxylin and eosin stained slides. Then each of the representative blocks was cut into consecutive $4 \mu \mathrm{m}$ thick slides. Firstly, the constructed slides were deparaffinized, rehydrated and heated for antigen retrieval. Secondly, the slides were incubated overnight with a primary antibody at $4^{\circ} \mathrm{C}$. The next day, the slides were incubated with the secondary antibody and stained with 3,3囚-diaminobenzidine. Appropriate positive and negative controls were used. The semi-quantification of Asporin and CD44 protein expression was performed independently by two pathologists according to previously published articles[13].

\section{Immunofluorescence staining}

Firstly, the samples were fixed in $4 \%$ paraformaldehyde; the cell membranes of samples were ruptured with $0.1 \%$ Triton X-100 and the samples were blocked with $3 \%$ BSA. Secondly, the samples were stained with primary antibody mixture at $4^{\circ} \mathrm{C}$ overnight. The next day, the samples were incubated with a secondary antibody mixture of rabbit anti-mouse-Alexa Fluor and goat anti-rabbit-FITC, mounted with Fluoromount G media with DAPI nuclear stain.

\section{Statistical analyses}

SPSS 17.0 was used to perform the statistical analyses. The associations between Asporin, CD44, Asporin/CD44 co-expression and the main clinicopathological factor were assessed by $\chi^{2}$ test. Kaplan-Meier method and log-rank test were used for survival analysis. The influence of various factors on survival was analyzed individually by univariate survival analysis. Variables with $P \leq 0.05$ in the univariate analysis were further analyzed using multivariate analysis.

\section{Results}

The clinicopathological characteristics of the 85 HGSOC patients were shown in Table 1. Firstly, we explored the expression and location of Asporin in 85 HGSOC patients.

\section{Asporin was mainly expressed in CAFs of 85 HGSOC patients, and high expression of Asporin was highly associated with chemoresistance.}

The IHC results showed that Asporin was mainly expression in cytoplasm of stromal cells adjacent to cancer cells in HGSOC tissues, while CD44 as a receptor of Asporin, was mainly expressed on membrane of tumor cells(Figure 1, 2). Further, we found that the expression pattern of Asporin was consistent with a-SMA, which is a specific CAFs biomarker (Figure 2). The positive rates of Asporin, CD44, Asporin /CD44 expression were 43.53\% (37/85), 34.12\% (29/85), 28.23\% (24/85) in all HGSOC tissues, $62.26 \%(33 / 53), 50.94 \%(27 / 53), 41.51 \%(22 / 53)$ in 53 chemo-resistance tissues(Figure 2) and 12.5\%(4/32), 6.25\% (2/32), 6.25\% (2/32) in chemosensitivity tissues respectively (Figure 3,Table 2) $(P<0.05)$.

\section{There were significant correlations between Asporin, CD44, Asporin/CD44 expression and CA125 level of blood, tumor differentiation, FIGO staging, chemoresistance of HGSOC patients.}

It is well known that chemoresistance, CA125 expression, tumor differentiation, FIGO staging and relapse are poor prognostic factors of HGSOC patients. Therefore, we further explored the association between Asporin, CD44, Asporin/CD44 expression and above clinicopathological characteristics of $85 \mathrm{HGSOC}$ patients. The results showed that Asporin, CD44, Asporin/CD44 expression were highly associated with chemoresistance, CA125 level of blood, tumor differentiation degree, FIGO staging and relapse of HGSOC patients $($ Table 2$)(P<0.05)$.

\section{High expression of Asporin, CD44 and co-expression of Asporin/CD44 were predictors for poor prognosis of HGSOC patients.}

The follow-up time of the enrolled HGSOC patients in our study was between 6 to 11 years. The median DFS was 9.8 months, and the median OS was 34.1 months. In addition to high CA125 expression, poor tumor differentiation, FIGO staging, chemo-resistance, P53 staining and Ki67 staining, univariate survival analyses revealed that Asporin, CD44, co-expression of Asporin/CD44 were also indicators for poor clinical outcome of HGSOC patients (Table 3). Further, multivariate Cox regression analysis revealed that positive expression of Asporin increased the hazard ratio 
of recurrence and death. Also, our results also showed that poorer tumor differentiation, more advanced FIGO stage and chemoresistance were independent indicators for unfavorable prognosis regarding both DFS and OS (Table 4).

\section{Discussion}

Ovarian cancer is the most deadly malignant female cancer, with a 5 -year survival less than $30 \%[1-2]$. Chemoresistance is one of the main reasons for the poor prognosis of ovarian cancer patients. Previous studies have shown that tumor microenvironment exserts huge potential in mediating the drug resistance of ovarian cancer [6-9]. It has been already proved that the research model of interactions between tumor microenvironment and ovarian cancer cells can more realistically simulate the environment of ovarian cancer in human body, providing new possibility to overcome ovarian cancer of human. However, the molecular mechanism of tumor microenvironment in chemresistance of human ovarian cancer still needs to be further explored. Recent years, the role of tumor microenvironment in human cancer has aroused our concern. Previous our research has demonstrated that CAFs of tumor microenvironment could promotes cancer cell invasion and migration in pancreatic cancer[13]. In the present study, by collecting the clinicopathological data of 53 platinum-resistant and 32 platinum-sensitive HGSOC patients, we demonstrated for the first time that Asporin, as a vital ECM protein of tumor microenvironment, was also mainly expressed in CAFs of tumor microenvironment and high expression of Asporin in CAFs indicated poor prognosis of HGSOC patients. In addition, we found that high expression of Asporin in CAFs was associated with chemoresistance of HGSOC patients, indicating that Asporin may be a promising biomarker to overcome platinum resistance of ovarian cancer.

CAF, as a major component of tumor microenvironment, plays a vital role in tumorigenesis and development[7]. The current research has found that CAFs could secrete a large number of cytokines, chemokines and growth factors to mediate ovarian cancer chemotherapy resistance, which is a hot area of ovarian cancer research at home and abroad in recent years [14-16]. Zhou B et al. found that CAFs promoted the cisplatin resistance of ovarian cancer cells by secreting CCL5 to activate STAT3 and PI3K/AKT signaling pathways[10]. The study of Deying W et al. found that CAFs could secrete hepatocyte growth factor which bind to the specific receptor c-Met, trigger the downstream PI3K/AKT pathway, and upregulate glucose regulatory protein to participate in the promotion of drug resistance in ovarian cancer[11]. Weimin W et al. found that CAFs could reduce the accumulation of cisplatin in ovarian cancer cells by releasing glutathione and cysteine, eventually leads to cisplatin resistance [9]. However, the molecular mechanism of CAFs-mediated chemotherapy resistance in ovarian cancer is far from being revealed. Therefore, our group has isolated and cultured CAFs from HGSOC patients for future research. Our previous study in pancreatic cancer also found that Asporin is mainly expressed in CAFs, and high expression of Asporin is associated with the poor prognosis of pancreatic cancer patients. In addition, our results also found that Asporin could interact with CD44 receptor of tumor cells and promote the invasion and migration of pancreatic cancer cells through activating ERK and AKT signaling pathways, which is coincident with the published studies[13]. Asporin belongs to the small leucine rich proteoglycan (SLRP) family. The earlier study found that Asporin played an important role in osteoarthritis, rheumatoid arthritis and lumbar disc degeneration and other bone and joint diseases by affecting the formation and differentiation of bone and cartilage[17-18]. Recently, some studies have confirmed that Asporin is secreted by CAFs and can promote the invasion of tumor cells by binding with CD44 receptor in a variety of human cancers $[13,19-20]$. Those studies suggest that Asporin could be a new potential target for human cancer treatment targeting the tumor microenvironment. HGSOC is the most common pathological subtype of ovarian cancer [2]. Nowadays, chemoresistance, CA125 level in blood, tumor differentiation degree, FIGO staging, p53 and Ki67 expression are definite factor in predicting prognosis of HGSOC patients. In our study, we demonstrated that Asporin is mainly expressed in CAFs of 85 HGSOC tissues by IHC and ICC, which was coincident with the published studies $[13,19]$. Our results also indicated that high expression of Asporin was associated with chemoresistance, as well as high expression of CA125 in blood, tumor poor differentiation, high FIGO staging, high expression of Ki67 and relapse of 85 HGSOC patients. In addition, high expression of Asporin indicated a worse prognosis of the HGSOC patients by Kaplan-Meier analysis, which was also consistent with previous researches[13,19]. Further, multivariate analyses indicated that Asporin was an independent factor for poor prognosis of HGSOC patients. Chemoresistance is one of the main reasons for the poor prognosis of HGSOC patients. Therefore, we speculated that Asporin may reduce survival time of HGSOC patients through enhancing drug resistance during chemotherapy. However, the exact mechanism still needs to be further explored in the future. CD44 as a membrane receptor for Asporin in many human cancers, plays an important role in the growth, recurrence and drug resistance of ovarian cancer[13,19]. We not only confirmed the expression and clinical value of CD44 in 85 HGSOC patients in the study, but also further demonstrated that Asporin/CD44 co-expression as well as tumor differentiation, FIGO staging, chemoresistance was an independent factor in predicting poor prognosis of HGSOC patients. Taking together, we preliminarily speculate that Asporin/CD44 axis may reduce the overall survival time of HGSOC patients by enhancing chemotherapy resistance during. However, the molecular mechanism will be further explored in our future research via the CAFs mode which we have successfully obtained from HGSOC patients.

In conclusion, high expression of Asporin was associated with high CA125 expression in blood, tumor poor differentiation, high FIGO staging, chemoresistance, high expression of Ki67 and relapse of HGSOC patients and was an independent indicator of poor prognosis of HGSOC patients.

\section{Abbreviations}


ASC: the American Cancer Society; HGSOC: High grade serous ovarian cancer; ECM: extracellular matrix; CAFs: Cancer-Associated-Fibroblasts; DFS: disease-free survival; OS: overall survival; FFPE: formalin fixed paraffin embedded; IRS: immunoreactive score; Cls: confidence intervals.

\section{Declarations}

\section{Ethics approval and consent to participate}

The Ethics Committee of the Qilu Hospital of Shandong University had approved the study. All living patients whose stored samples were used in the study had acquired written consent.

\section{Consent for publication}

Not applicable.

\section{Availability of data and material}

All data supporting the paper is included in the article.

\section{Competing interests}

The authors declare that they have no competing interests.

\section{Funding}

This work was supported by the findings from the National Natural Science Foundation of China (82002733), Shandong Medical and Health Science and Technology Development Plan (2018WS330,2018WS279).

\section{Authors' contributions}

The study was conceived and designed by LJ. The whole experiment was performed by LW and XZ. The statistical analysis was conducted by LW and JZ. The manuscript was written by LW, YH and WZ. All authors read and approved the manuscript.

\section{Acknowledgements}

We thank gynecology oncology key laboratory of qilu hospital for their detailed clinical and follow-up data.

\section{References}

1. Bookman MA. Optimal primary therapy of ovarian cancer. Ann Oncol. 2016;27 Suppl 1:i58-i62. doi:10.1093/annonc/mdw088.

2. Siegel RL, Miller KD, Fuchs HE, Jemal A. Cancer Statistics, 2021 [published correction appears in CA Cancer J Clin. 2021 Jul;71(4):359]. CA Cancer J Clin. 2021;71(1):7-33. doi:10.3322/caac.21654.

3. Bu H, Li Y, Jin C, et al. Overexpression of PRC1 indicates a poor prognosis in ovarian cancer. Int J Oncol. 2020;56(3):685-696. doi:10.3892/ijo.2020.4959

4. Yao Q, Qu X, Yang Q, et al. Blockage of transdifferentiation from fibroblast to myofibroblast in experimental ovarian cancer models [retracted in: Mol Cancer. 2009;8:84]. Mol Cancer. 2009;8:78. Published 2009 Sep 27. doi:10.1186/1476-4598-8-78.

5. Wu H, Li R, Zhang Z, et al. Kallistatin inhibits tumour progression and platinum resistance in high-grade serous ovarian cancer [published correction appears in J Ovarian Res. 2020 Mar 13;13(1):28]. J Ovarian Res. 2019;12(1):125. Published 2019 Dec 29. doi:10.1186/s13048019-0601-6.

6. Luo Z, Wang Q, Lau WB, et al. Tumor microenvironment: The culprit for ovarian cancer metastasis?. Cancer Lett. 2016;377(2):174-182. doi:10.1016/j.canlet.2016.04.038.

7. Hui L, Chen Y. Tumor microenvironment: Sanctuary of the devil. Cancer Lett. 2015;368(1):7-13. doi:10.1016/j.canlet.2015.07.039.

8. Worzfeld T, Pogge von Strandmann E, Huber M, et al. The Unique Molecular and Cellular Microenvironment of Ovarian Cancer. Front Oncol. 2017;7:24. Published 2017 Feb 22. doi:10.3389/fonc.2017.00024.

9. Wang W, Kryczek I, Dostál L, et al. Effector T Cells Abrogate Stroma-Mediated Chemoresistance in Ovarian Cancer. Cell. 2016;165(5):10921105. doi:10.1016/j.cell.2016.04.009.

10. Zhou B, Sun C, Li N, et al. Cisplatin-induced CCL5 secretion from CAFs promotes cisplatin-resistance in ovarian cancer via regulation of the STAT3 and PI3K/Akt signaling pathways. Int J Oncol. 2016;48(5):2087-2097. doi:10.3892/ijo.2016.3442. 
11. Deying W, Feng G, Shumei L, Hui Z, Ming L, Hongqing W. CAF-derived HGF promotes cell proliferation and drug resistance by up-regulating the c-Met/PI3K/Akt and GRP78 signalling in ovarian cancer cells. Biosci Rep. 2017;37(2):BSR20160470. Published 2017 Apr 10. doi:10.1042/BSR20160470

12. Santi A, Kugeratski FG, Zanivan S. Cancer Associated Fibroblasts: The Architects of Stroma Remodeling. Proteomics. 2018;18(56):e1700167. doi:10.1002/pmic.201700167

13. Wang L, Wu H, Wang L, et al. Asporin promotes pancreatic cancer cell invasion and migration by regulating the epithelial-to-mesenchymal transition (EMT) through both autocrine and paracrine mechanisms. Cancer Lett. 2017;398:24-36. doi:10.1016/j.canlet.2017.04.001

14. Hurley PJ, Sundi D, Shinder B, et al. Germline Variants in Asporin Vary by Race, Modulate the Tumor Microenvironment, and Are Differentially Associated with Metastatic Prostate Cancer. Clin Cancer Res. 2016;22(2):448-458. doi:10.1158/1078-0432.CCR-15-0256.

15. Turtoi A, Musmeci D, Wang $Y$, et al. Identification of novel accessible proteins bearing diagnostic and therapeutic potential in human pancreatic ductal adenocarcinoma. J Proteome Res. 2011;10(9):4302-4313. doi:10.1021/pr200527z.

16. Maris P, Blomme A, Palacios AP, et al. Asporin Is a Fibroblast-Derived TGF- $\beta 1$ Inhibitor and a Tumor Suppressor Associated with Good Prognosis in Breast Cancer. PLoS Med. 2015;12(9):e1001871. Published 2015 Sep 1. doi:10.1371/journal.pmed.1001871.

17. Yamada S, Murakami S, Matoba R, et al. Expression profile of active genes in human periodontal ligament and isolation of PLAP-1, a novel SLRP family gene. Gene. 2001;275(2):279-286. doi:10.1016/s0378-1119(01)00683-7.

18. Nakajima M, Kizawa H, Saitoh M, Kou I, Miyazono K, lkegawa S. Mechanisms for asporin function and regulation in articular cartilage. $J$ Biol Chem. 2007;282(44):32185-32192. doi:10.1074/jbc.M700522200.

19. Satoyoshi R, Kuriyama S, Aiba N, Yashiro M, Tanaka M. Asporin activates coordinated invasion of scirrhous gastric cancer and cancerassociated fibroblasts. Oncogene. 2015;34(5):650-660. doi:10.1038/onc.2013.584.

20. Sasaki Y, Takagane K, Konno T, et al. Expression of asporin reprograms cancer cells to acquire resistance to oxidative stress. Cancer Sci. 2021;112(3):1251-1261. doi:10.1111/cas.14794.

\section{Tables}

Table 1. Clinical and pathological characteristics of 85 HGSOC patients. 


\begin{tabular}{|c|c|}
\hline Parameter & No. of patients(\%)Number(\%) \\
\hline \multicolumn{2}{|l|}{ Age at diagnosis } \\
\hline$\leq 50$ & $24(28.24 \%)$ \\
\hline$\otimes 50$ & $61(71.76 \%)$ \\
\hline \multicolumn{2}{|c|}{ Neoadjuvant chemotherapy } \\
\hline Yes & $38(44.71 \%)$ \\
\hline No & $47(55.29 \%)$ \\
\hline \multicolumn{2}{|l|}{ CA125 } \\
\hline$\leq 35$ & $14(16.47 \%)$ \\
\hline$凶 35$ & $71(83.53 \%)$ \\
\hline \multicolumn{2}{|c|}{ Number of deliveries } \\
\hline$\leq 2$ & $39(45.88 \%)$ \\
\hline$凶 2$ & $46(52.12 \%)$ \\
\hline \multicolumn{2}{|l|}{ Menopausal age } \\
\hline$\leq 50$ & $28(32.94 \%)$ \\
\hline$\varangle 50$ & $57(67.06 \%)$ \\
\hline \multicolumn{2}{|l|}{ Complications } \\
\hline Yes & $54(63.53 \%)$ \\
\hline No & $31(36.47 \%)$ \\
\hline \multicolumn{2}{|c|}{ Degree of differentiation } \\
\hline Low & $75(88.24 \%)$ \\
\hline Moderate/high & $10(11.76 \%)$ \\
\hline \multicolumn{2}{|l|}{ FIGO staging } \\
\hline $1 / I I$ & $17(20.00 \%)$ \\
\hline III/IV & $68(80.00 \%)$ \\
\hline \multicolumn{2}{|l|}{ Chemo-resistance } \\
\hline Yes & $45(52.94 \%)$ \\
\hline No & $40(47.06 \%)$ \\
\hline \multicolumn{2}{|l|}{ P53 staining } \\
\hline High expression & $24(28.24 \%)$ \\
\hline Low expression & $61(71.16 \%)$ \\
\hline \multicolumn{2}{|l|}{ Ki67 staining } \\
\hline High expression & $58(68.24 \%)$ \\
\hline Low expression & $27(31.76 \%)$ \\
\hline \multicolumn{2}{|l|}{ Relapse } \\
\hline Yes & $13(15.29 \%)$ \\
\hline No & $72(84.71 \%)$ \\
\hline
\end{tabular}

Table 2. Correlation between Asporin, CD44, Asporin/CD44 expression and clinicopathological characteristics of 85 HGSOC patients. 


\begin{tabular}{|c|c|c|c|c|c|c|c|c|c|c|}
\hline \multirow[b]{2}{*}{ Parameter } & \multirow[b]{2}{*}{ Cases } & \multicolumn{2}{|l|}{ Asporin $\mathrm{n}(\%)$} & \multirow[t]{2}{*}{$P$} & \multicolumn{2}{|l|}{ CD44 n (\%) } & \multirow[t]{2}{*}{$\mathbf{P}$} & \multicolumn{2}{|c|}{ Asporin/CD44 n (\%) } & \multirow[t]{2}{*}{$P$} \\
\hline & & Negative & Positive & & Negative & Positive & & Negative & Positive & \\
\hline Age at & & & & 0.395 & & & 0.800 & & & 0.195 \\
\hline - 50 . & 24 & $15(62.50 \%)$ & $9(37.50 \%)$ & & $16(66.67 \%)$ & $8(33.330 \%)$ & & $17(70.83 \%)$ & $7(29.17 \%)$ & \\
\hline$=0$ & 61 & $33(54.10 \%)$ & $28(45.90 \%)$ & & $40(65.57 \%)$ & $21(34.43 \%)$ & & $44(72.13 \%)$ & $17(27.8 \%)$ & \\
\hline Neo- & & & & 0.285 & & & 0.166 & & & 0.039 \\
\hline Yoa & 38 & $19(50.00 \%)$ & $19(50.00 \%)$ & & $22(57.89 \%)$ & $16(42.11 \%)$ & & $23(60.53 \%)$ & $15(39.47 \%)$ & \\
\hline No & 47 & $29(61.70 \%)$ & $18(38.30 \%)$ & & $34(72.34 \%)$ & $13(27.66 \%)$ & & $38(80.85 \%)$ & $9(19.15 \%)$ & \\
\hline CA125 & & & & 0.007 & & & 0.008 & & & 0.004 \\
\hline$\leq 35$ & 14 & $9(64.29 \%)$ & $5(35.71 \%)$ & & $11(78.57 \%)$ & $3(21.42 \%)$ & & $12(85.71 \%)$ & $2(14.29 \%)$ & \\
\hline$凶 35$ & 71 & $39(54.93 \%)$ & $32(45.07 \%)$ & & $45(63.38 \%)$ & $26(36.62 \%)$ & & $49(69.01 \%)$ & $22(30.99 \%)$ & \\
\hline Number of & & & & 0.141 & & & 0.030 & & & 0.014 \\
\hline-2 & 41 & $25(60.98 \%)$ & $16(39.02 \%)$ & & $32(78.05 \%)$ & $9(21.95 \%)$ & & $34(82.93 \%)$ & $7(17.07 \%)$ & \\
\hline$\otimes 2$ & 44 & $23(52.27 \%)$ & $21(47.73 \%)$ & & $24(54.54 \%)$ & $20(45.45 \%)$ & & $27(61.36 \%)$ & $17(38.64 \%)$ & \\
\hline Menopausal & & & & 0.395 & & & 0.800 & & & 0.195 \\
\hline & 28 & $15(53.57 \%)$ & $13(46.43 \%)$ & & $16(57.14 \%)$ & $12(42.86 \%)$ & & $17(60.71 \%)$ & $11(39.28 \%)$ & \\
\hline 850 & 57 & $33(57.89 \%)$ & $24(42.11 \%)$ & & $40(70.18 \%)$ & $17(29.82 \%)$ & & $44(77.19 \%)$ & $13(22.81 \%)$ & \\
\hline Complications & & & & 0.769 & & & 0.038 & & & 0.156 \\
\hline Yes & 54 & $30(55.56 \%)$ & $24(44.44 \%)$ & & $31(57.41 \%)$ & $23(52.59 \%)$ & & $36(66.67 \%)$ & 18(33.33\%) & \\
\hline No & 31 & $18(58.06 \%)$ & $13(41.94 \%)$ & & $25(80.65 \%)$ & $6(19.35 \%)$ & & $25(80.65 \%)$ & $6(19.35 \%)$ & \\
\hline Differentiation & & & & 0.023 & & & 0.040 & & & 0.035 \\
\hline Low & 75 & $39(52.00 \%)$ & $36(48.00 \%)$ & & $47(62.67 \%)$ & 28(37.33\%) & & $51(68.00 \%)$ & $24(32.00 \%)$ & \\
\hline Moderate/high & 10 & $9(90.00 \%)$ & $1(10.00 \%)$ & & $9(90.00 \%)$ & $1(10.00 \%)$ & & $10(100.0 \%)$ & $0(00.0 \%)$ & \\
\hline FIGO staging & & & & 0.003 & & & 0.006 & & & 0.003 \\
\hline $1 / I I$ & 17 & $15(88.26 \%)$ & $2(11.76 \%)$ & & $16(94.12 \%)$ & $1(5.88 \%)$ & & $12(70.59 \%)$ & $5(29.41 \%)$ & \\
\hline III/IV & 68 & $33(48.53 \%)$ & $35(51.47 \%)$ & & $40(58.82 \%)$ & $28(41.18 \%)$ & & $49(72.06 \%)$ & $19(27.94 \%)$ & \\
\hline Chemo- & & & & 0.019 & & & 0.016 & & & 0.013 \\
\hline testa & 53 & $20(37.74 \%)$ & $33(62.26 \%)$ & & $26(49.06 \%)$ & $27(50.94 \%)$ & & $31(58.49 \%)$ & $22(41.51 \%)$ & \\
\hline (c) & 32 & $28(87.5 \%)$ & $4(12.5 \%)$ & & $30(93.75 \%)$ & $2(6.25 \%)$ & & $30(93.75 \%)$ & $2(6.25 \%)$ & \\
\hline No & & & & 0.096 & & & 0.271 & & & 0.348 \\
\hline 200 & 24 & $17(70.83 \%)$ & $7(29.17 \%)$ & & $19(79.17 \%)$ & $5(20.83 \%)$ & & $19(54.17 \%)$ & $5(54.17 \%)$ & \\
\hline expression & 61 & $31(50.82 \%)$ & $30(49.18 \%)$ & & $37(60.66 \%)$ & $\begin{array}{l}24 \\
(39.34 \%)\end{array}$ & & $42(68.85 \%)$ & $19(31.15 \%)$ & \\
\hline $\begin{array}{l}\text { Low } \\
\text { exprescion }\end{array}$ & & & & 0.001 & & & 0.01 & & & 0.001 \\
\hline 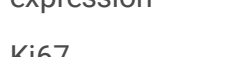 & 58 & $25(70.83 \%)$ & $33(29.17 \%)$ & & $33(57.00 \%)$ & (1) & & $35(60.34 \%)$ & $23(39.66 \%)$ & \\
\hline & 27 & 23(85.19\%) & $4(14.81 \%)$ & & $23(85.19 \%)$ & $20(40.00$ & & $26(96.30 \%)$ & $1(3.70 \%)$ & \\
\hline $\begin{array}{l}\text { High } \\
\text { expression }\end{array}$ & & & & 0.000 & & $4(14.81 \%)$ & 0.029 & & & 0.014 \\
\hline Low & 72 & $35(48.61 \%)$ & $37(51.39 \%)$ & & $44(61.11 \%)$ & & & $48(66.67 \%)$ & $24(33.33 \%)$ & \\
\hline & 13 & $13(100.0 \%)$ & $0(00.00 \%)$ & & $12(92.31 \%)$ & $28(38.89 \%)$ & & $13(100.0 \%)$ & $0(0.00 \%)$ & \\
\hline
\end{tabular}


Table 3. Univariate analyses for DFS and OS. 


\begin{tabular}{|c|c|c|c|c|}
\hline \multirow[b]{2}{*}{ Variable } & \multicolumn{2}{|l|}{ DFS } & \multicolumn{2}{|l|}{ OS } \\
\hline & Median survival(months) & $\mathrm{P}$ & Median survival(months) & $P$ \\
\hline Age at diagnosis & & 0.440 & & 0.767 \\
\hline$\leq 50$ & 12.00 & & 35.00 & \\
\hline$\otimes 50$ & 10.00 & & 33.00 & \\
\hline Neo-chemotherapy & & 0.135 & & 0.178 \\
\hline Yes & 13.00 & & 34.00 & \\
\hline No & 9.00 & & 34.00 & \\
\hline CA125 & & 0.005 & & 0.039 \\
\hline$\leq 35$ & 15.00 & & 40.00 & \\
\hline$\otimes 35$ & 7.00 & & 23.00 & \\
\hline Number of deliveries & & 0.157 & & 0.101 \\
\hline$\leq 2$ & 13.00 & & 33.00 & \\
\hline$凶 2$ & 11.00 & & 36.00 & \\
\hline Differentiation & & 0.001 & & 0.002 \\
\hline Low & 6.00 & & 19.00 & \\
\hline Moderate/high & 17.00 & & 46.00 & \\
\hline Menopausal age & & 0.394 & & 0.094 \\
\hline$\leq 50$ & 13.00 & & 36.00 & \\
\hline$\otimes 50$ & 10.00 & & 32.00 & \\
\hline FIGO staging & & 0.001 & & 0.000 \\
\hline $1 / I I$ & 15.00 & & 45.00 & \\
\hline III/IV & 5.00 & & 12.00 & \\
\hline Chemo-resistance & & 0.001 & & 0.000 \\
\hline Yes & 7.00 & & 44.00 & \\
\hline No & 14.00 & & 13.00 & \\
\hline P53 staining & & 0.001 & & 0.001 \\
\hline High expression & 15.00 & & 43.00 & \\
\hline Low expression & 7.00 & & 12.00 & \\
\hline Ki67 staining & & 0.005 & & 0.000 \\
\hline High expression & 6.00 & & 11.00 & \\
\hline Low expression & 14.00 & & 46.00 & \\
\hline Asporin expression & & 0.001 & & 0.001 \\
\hline High expression & 5.00 & & 44.00 & \\
\hline Low expression & 16.00 & & 15.00 & \\
\hline CD44 expression & & 0.021 & & 0.010 \\
\hline High expression & 6.00 & & 16.00 & \\
\hline Low expression & 15.00 & & 46.00 & \\
\hline Asporin/CD44 co-exp & & & & \\
\hline High expression & & 0.017 & & 0.001 \\
\hline Low expression & 15.00 & & 15.00 & \\
\hline
\end{tabular}

Page 10/13 
Table 4. Multivariate analyses for DFS and OS.

\begin{tabular}{|c|c|c|}
\hline Parameter & $\mathrm{HR}(95 \% \mathrm{Cl})$ & $\mathbf{P}$ \\
\hline \multicolumn{3}{|c|}{ DFS: Cox regression model } \\
\hline FIGO staging & & 0.003 \\
\hline \multicolumn{3}{|l|}{$\mathrm{I} / \mathrm{II}$} \\
\hline III/IV & 8.708(2.900-26.153) & \\
\hline Differentiation & & 0.005 \\
\hline \multicolumn{3}{|l|}{ Low } \\
\hline Moderate/high & 4.191(2.458-7.934) & \\
\hline Chemo-resistance & & 0.000 \\
\hline \multicolumn{3}{|l|}{ Yes } \\
\hline No & 93.022(11.899-727.228) & \\
\hline Asporin expression & & 0.007 \\
\hline \multicolumn{3}{|l|}{ High expression } \\
\hline Low expression & 2.044(1.216-3.435) & \\
\hline \multicolumn{3}{|c|}{ Asporin/CD44 co-expression } \\
\hline High expression & & 0.002 \\
\hline \multicolumn{3}{|l|}{ Low expression } \\
\hline & $3.324(2.112-5.231)$ & \\
\hline \multicolumn{3}{|c|}{ OS: Cox regression model } \\
\hline FIGO staging & & 0.002 \\
\hline \multicolumn{3}{|l|}{$\mathrm{I} / \mathrm{II}$} \\
\hline III/IV & $6.431(2.002-20.655)$ & \\
\hline Differentiation & & 0.000 \\
\hline \multicolumn{3}{|l|}{ Low } \\
\hline Moderate/high & $6.527(2.034-12.031)$ & \\
\hline \multicolumn{3}{|l|}{ Chemo-resistance } \\
\hline Yes & & 0.000 \\
\hline No & $3.484(1.901-6.386)$ & \\
\hline Asporin expression & & 0.048 \\
\hline \multicolumn{3}{|l|}{ High expression } \\
\hline Low expression & $2.139(1.006-4.551)$ & \\
\hline \multicolumn{3}{|c|}{ Asporin/CD44 co-expression } \\
\hline High expression & & 0.000 \\
\hline \multicolumn{3}{|l|}{ Low expression } \\
\hline & $7.288(3.229-16.452)$ & \\
\hline
\end{tabular}

\section{Figures}




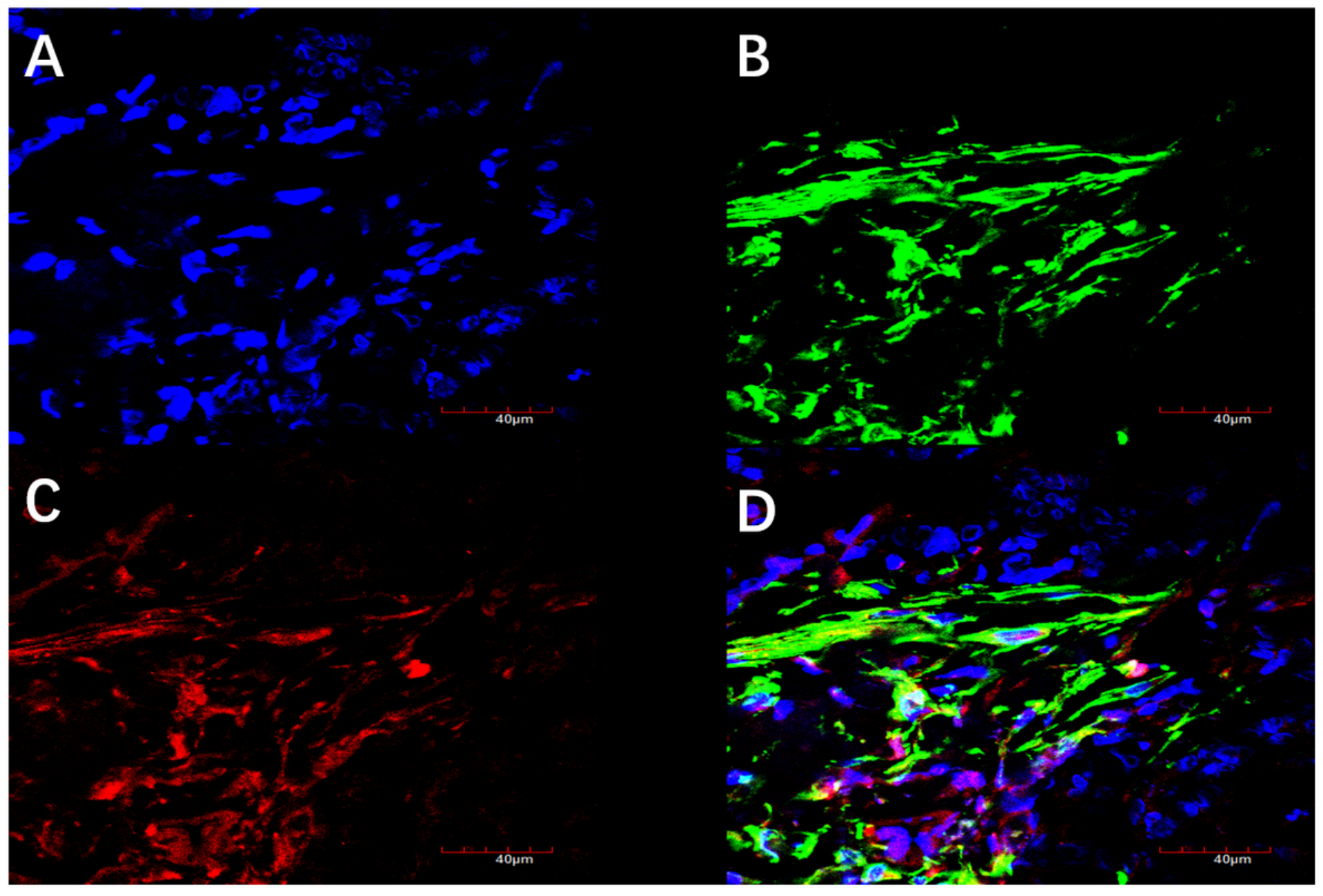

Figure 1

Expression and location of Asporin by immunofluorescence staining. Asporin is mainly highly expressed in the interstitium of ovarian cancer, which is consistent with the expression of a-SMA. Cell nucleus (A); a-SMA(B); Asporin(C); Merge(D). Manification x 100.

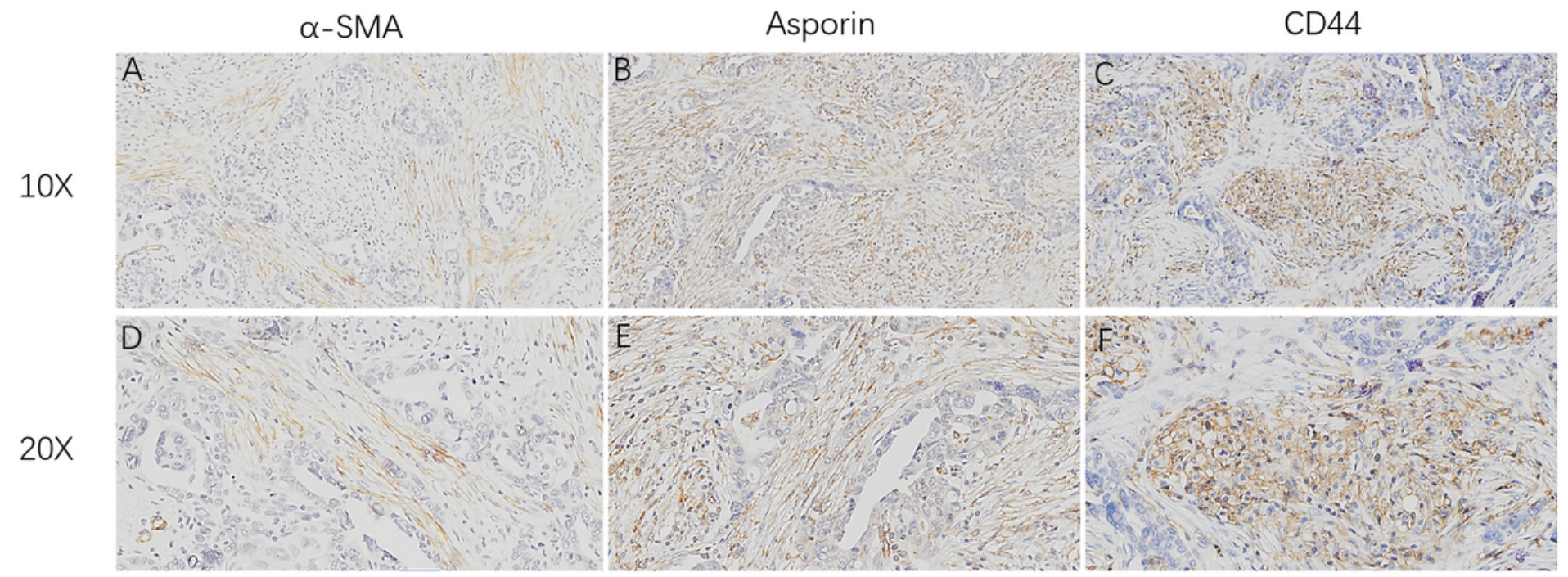

Figure 2

Asporin was mainly expression in cytoplasm of stromal cells adjacent to cancer cells in chemo-resistance HGSOC tissues, while CD44 was mainly expressed on membrane of tumor cells. In addition, Asporin was consistent with a-SMA, which is a specific CAFs biomarker. a-SMA(A and 
D); Asporin(B and E); CD44(C and F).

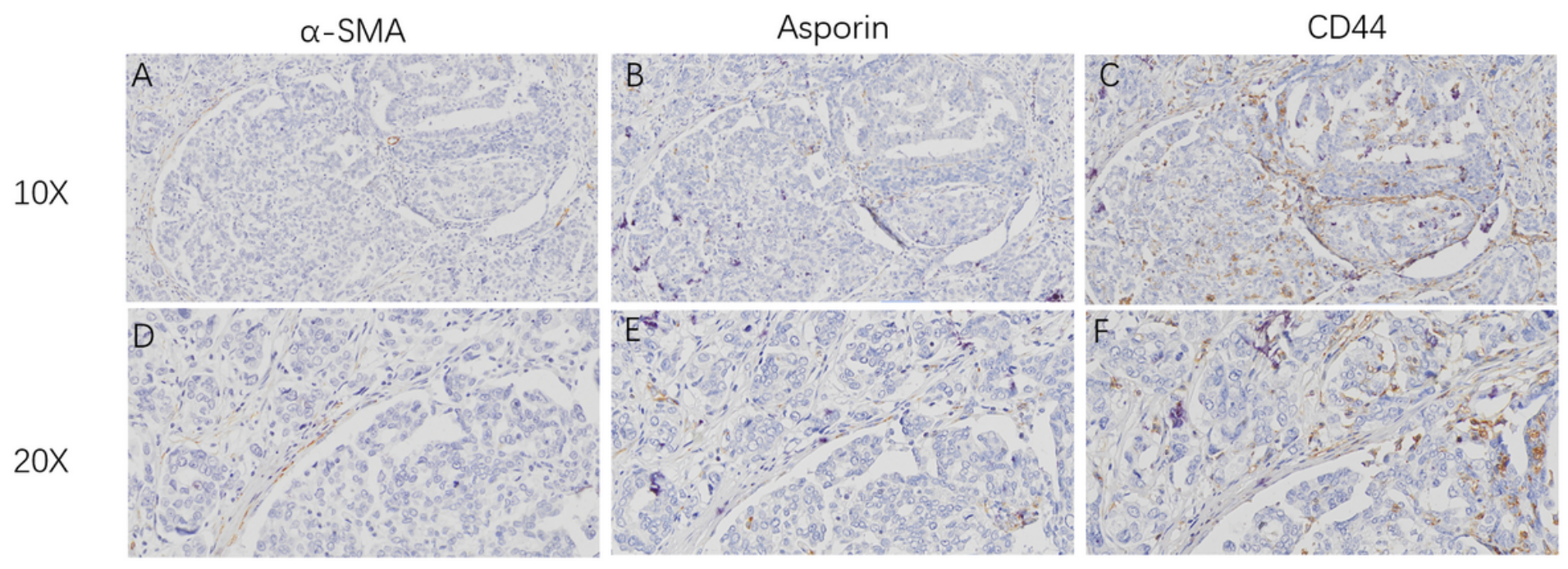

\section{Figure 3}

Asporin and CD44 was mainly low expressed in chemosensitivity HGSOC tissued. a-SMA(A and D); Asporin(B and E); CD44(C and F).
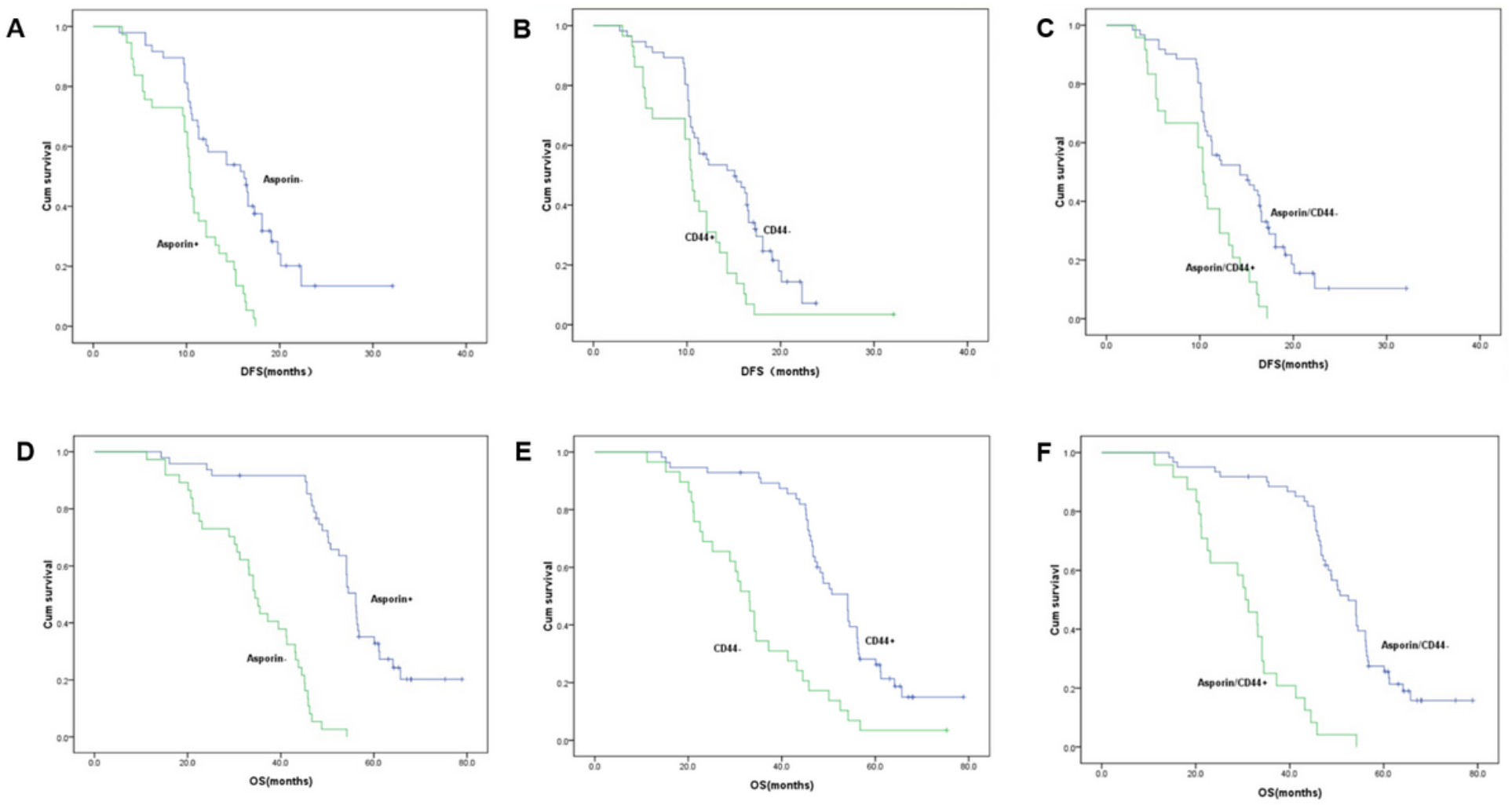

\section{Figure 4}

Kaplan-Meier curves of DFS and OS with different expression of Asporin and CD44. DFS according to Asporin expression pattern ( $\mathrm{P}=0.021)(\mathrm{A})$, CD44 expression pattern $(P=0.016)(B)$ and Asporin/CD44 co-expression pattern $(P=0.023)(C)$. OS according to Asporin expression pattern $(P=$ $0.011)(E), C D 44$ expression pattern $(P=0.032)(F)$ and Asporin/CD44 co-expression pattern $(P=0.001)$. 